\title{
Small-signal Stability Boundary of Heterogenous Multi- converter Power Systems Dominated by Phase-locked Loop Dynamics
}

This paper was downloaded from TechRxiv (https://www.techrxiv.org).

\section{LICENSE}

CC BY-NC-SA 4.0

SUBMISSION DATE / POSTED DATE

$17-01-2022$ / 20-01-2022

\section{CITATION}

Zhou, Yuhan; Xin, Huanhai; Wu, Di; Wang, Guanzhong; Yuan, Hui; Ju, Ping (2022): Small-signal Stability Boundary of Heterogenous Multi-converter Power Systems Dominated by Phase-locked Loop Dynamics. TechRxiv. Preprint. https://doi.org/10.36227/techrxiv.18517835.v1

$\mathrm{DOI}$ 


\title{
Small-signal Stability Boundary of Heterogeneous Multi-converter Power Systems Dominated by Phase- locked Loop Dynamics
}

\author{
Yuhan Zhou, Huanhai Xin*, Di Wu, Guanzhong Wang, Hui Yuan, Ping Ju
}

\begin{abstract}
While renewable resources are increasingly integrated into the electric power grid, the small-signal instability risk may be induced by grid-following converters using phaselocked loops (PLLs) for grid synchronization, especially under weak grid conditions. The analysis of the instability mechanism is complex in a multi-converter system due to the dynamic interaction between PLL-based converters and the power network. The analysis complexity is further increased in a heterogeneous multi-converter system (HMCS), where all converters have different control configurations and parameters from different manufacturers. To understand how the different PLL dynamics collectively affect the stability of the HMCS, this paper analytically derives the small-signal stability boundary condition of the HMCS dominated by the PLL dynamics (HMCS-DPLL). The derived stability boundary allows us to obtain analytical results about how the stability of the HMCS-DPLL is affected by grid strength, converter operating conditions, different PLL control parameters and the interaction among different PLLs. Based on the stability boundary condition, a computationally efficient method is also proposed to identify the design rationality of PLL control parameters as well as the small-signal stability and stability margin of the HMCS-DPLL. The analytical results and proposed method are validated by modal analysis and electromagnetic transient simulation with detailed models on a 9-converter heterogeneous system.
\end{abstract}

Index Terms-PLLs, heterogeneous multi-converter system, stability boundary condition.

\section{INTRODUCTION}

The growing number of renewable resources such as wind and solar are usually being integrated into the power grid through grid-following converters using the phase-locked loops (PLLs) for grid synchronization [1]-[5]. It has been indicated that the small-signal instability risk may be induced in the power system by the PLL dynamics, especially under the weak grid connections [1]-[9]. Hence, it is essential to investigate and understand how instability risk is introduced into the power system by the multiple PLLs, which is useful to guide the grid planning to reduce and even mitigate the instability risk in the multi-converter system.

In the previous study, the instability analysis was mainly investigated in a grid-connected converter system caused by a single PLL, and the analysis techniques mainly include modal analysis method, impedance-based analysis method, and openloop mode resonance method, etc. The modal analysis method in [7]-[9] demonstrated that when the network connection condition becomes weak or the setting of the PLL PI parameter

Y. Zhou, H. Xin, G Wang, H. Yuan, P. Ju are with the college of electrical engineering, Zhejiang University, Hangzhou 310027, China (Email: xinhh@zju.edu.cn; zhouyuhan@zju.edu.cn). is improper, the oscillation modes related to the PLL may move toward the right-half complex plane or system may become instable. However, the modal analysis method could not explain the instability mechanism caused by the PLL dynamics. To this end, the impedance-based analysis method in [10]-[12] considers negative effective resistance of the converter as the potential oscillation source caused by the PLL dynamics. Particularly, when PLL bandwidth increases or grid strength weakens, the negative resistance effect is further exacerbated, which may increase the instability risk of the system. In [13][15], the mechanism investigated by open-loop mode resonance method manifested that when the bandwidth of the PLL is similar to that of the outer- or inner-loop, the strong dynamic interaction may exist between the two control links, which may also deteriorate the oscillation modes corresponding to the PLLs. However, the above analysis method may be inapplicable in the multi-converter system. It is challenging to analyze the instability mechanism caused by multiple PLLs in a multi-converters system due to the complex interaction among converters interconnected through the power network.

To address the above challenge, several existing solutions have been proposed in the stability analysis of multi-converter systems. In [16]-[17], the capacity-weighted mean value method was proposed to aggregate a multi-converter system into an equivalent single-converter model. Essentially, this analysis method still investigates the influence of a single PLL on the system stability, which does not fully consider the dynamic interaction among different PLLs of converters. Instead of the aggregated converter model, a method was proposed in [18]-[19] by decoupling a homogeneous multiconverter system into a set of equivalent single-converter system. On this basis, the small-signal stability analysis experience for a single PLL can be easily transplanted to the multi-converter system with multiple PLLs, though this study does not provide analytical results and conclusions applicable to the PLL stability mechanism analysis. Following the idea, studies in [20] analytically derived the stability limit of a homogeneous multi-converter system dominated by the dynamics of multiple PLLs, which can be conducted by a simplified multi-converter model only including the dynamics of PLLs and power network. A practical power system is usually a heterogeneous multi-converter system (HMCS), since converters may come from different manufacturers equipped with different PLL control parameters. In such a heterogeneous

Di Wu is with the department of electrical and computer engineering, North Dakota State University, Fargo, 58102, USA. 
system, the interaction among the interconnected converters and power network become more complex than those in a homogeneous system. Hence, it is urgent to explore the instability mechanism induced by the PLLs with different control parameters and the stability analysis methods in the HMCS.

This paper analytically derives the small-signal stability boundary of the HMCS dominated by the PLL dynamics (HMCS-DPLL) to explore the insights into how the PLL dynamics collectively affect the stability of the HMCS. To this end, we first construct a reduced-order model of a HMCSDPLL mainly including the dynamics of different PLLs and power network. Then, it is theoretically proved that the reduced HMCS-DPLL can be converted into an equivalent homogeneous system in order to decouple it into a set of equivalent single-converter subsystems for the stability analysis. Thus, the small-signal stability of the HMCS-DPLL can be equivalently characterized by the most critical decoupled subsystem. On this basis, the stability boundary condition of the HMCS-DPLL is analytically derived. The derived stability boundary can be used to explore insights into the instability mechanism. Based on the stability boundary, a method is proposed to identify the design rationality of PLL control parameters as well as the small-signal stability and stability margin of the HMCS-DPLL. The derived stability boundary and the proposed method will be useful for grid planning as well as PLL control design to reduce or mitigate the instability risk in the HMCS-DPLL at the planning stage. The major contributions of this paper can be summarized as follows:

1) Based on modal decoupling theory and matrix perturbation theory, it is theoretically proved that the HMCS-DPLL can be equivalently converted into the most critical decoupled subsystem for small-signal stability analysis. On this basis, the instability mechanism induced by the PLL in the equivalent single-converter system can be transplanted to the heterogeneous multi-converter system, thus significantly reducing the complexity of the small-signal stability analysis of a HMCS-DPLL.

2) The derived stability boundary condition can explore insights into the instability mechanism of the HMCSDPLL and explain how the stability of the HMCS-DPLL is affected by network connection, converter operating conditions, PLL control parameters, and dynamic interaction among different PLLs.

3) Based on the derived boundary condition, a method is proposed to identify the small-signal stability and stability margin of the HMCS-DPLL as well as the design rationality of PLL control parameters. The proposed method is computationally efficient because it does not need to establish a detailed full-order model, and avoid high-order modal analysis process, which significantly increases the engineering applicability of the proposed method.

4) The derived stability boundary condition, the analytical conclusions, and the proposed method are validated by modal analysis and electromagnetic transient simulation with detailed models on a 9-converter heterogeneous system.

The rest of this paper is organized as follows. In Section II the full-order HMCS model and the reduced-order HMCSDPLL model are presented. In Section III, the small-signal stability of the HMCS-DPLL is analyzed, and the stability boundary condition for the HMCS-DPLL is derived. On this basis, the instability mechanism in the HMCS-DPLL is explored. In Section IV, the efficacy of the derived stability boundary condition, the analytical results, and the proposed method is demonstrated by modal analysis and electromagnetic transient simulation on a 9-converter heterogeneous system. In Section V, the conclusions are drawn.

\section{II.MODELING OF HMCS-DPLL}

\section{A. Full-order modeling of HMCS}

Let us consider a HMCS with $n$ converters. The system can be modelled by two parts: the converters and power network. The dynamics of the $i^{\text {th }}$ converter can be characterized by the following state-space model:

$$
\left\{\begin{array}{l}
\frac{d}{d t} \Delta \boldsymbol{X}_{i}=\boldsymbol{A}_{i} \Delta \boldsymbol{X}_{i}+\boldsymbol{B}_{i} \Delta \boldsymbol{U}_{i} \\
\Delta \boldsymbol{I}_{i}=\boldsymbol{C}_{i} \Delta \boldsymbol{X}_{i}
\end{array} \quad i=1, \cdots, n\right.
$$

where $\boldsymbol{I}_{i}=\left[I_{x, i} I_{y, i}\right]^{T}$ and $\boldsymbol{U}_{i}=\left[U_{x, i} U_{y, i}\right]^{T}$ are the output current and terminal voltage of the $i^{\text {th }}$ converter expressed in the common $x-y$ coordinate; $\boldsymbol{X}_{i}$ denotes a column vector including all the state variables of the $i^{\text {th }}$ converter; $\boldsymbol{A}_{i}, \boldsymbol{B}_{i}$ and $\boldsymbol{C}_{i}$ are state matrix, control matrix, and output matrix of the $i^{\text {th }}$ converter under the capacity base of the system, respectively; $\Delta$ denotes the small increment of a vector.

The dynamics model of the power network in the HMCS can be represented by:

$$
\Delta \boldsymbol{U}=(\boldsymbol{Z} \otimes \boldsymbol{\alpha}(s)) \Delta \boldsymbol{I}, \boldsymbol{\alpha}(s)=\left[\begin{array}{cc}
(s+\tau) / \omega_{0} & -1 \\
1 & (s+\tau) / \omega_{0}
\end{array}\right]
$$

where $\Delta \boldsymbol{U}=\left[\Delta \boldsymbol{U}_{1}{ }^{\mathrm{T}}, \ldots, \Delta \boldsymbol{U}_{n}{ }^{\mathrm{T}}\right] ; \Delta \boldsymbol{I}=\left[\Delta \boldsymbol{I}_{1}^{\mathrm{T}}, \ldots, \Delta \boldsymbol{I}_{n}^{\mathrm{T}}\right] ; \boldsymbol{Z}$ is Kronreduced reactance matrix only including $n$ converter nodes; $s$ represents Laplace operator; $\gamma_{0}$ is the rated angular velocity of the ac system; $\tau$ is the ratio of the line resistance to line inductance (i.e., $\ddot{U}=R / L$ ) in the power network, and it is a constant value for a given power network; and symbol $\hat{\vartheta}$ denotes the Kronecker product.

\section{B. Reduced-order modeling of HMCS-DPLL}

To focus on the small-signal stability of the HMCS dominated by the dynamics of the PLLs, a reduced-order converter model describing the dynamics of PLLs is derived in this section. The commonly used synchronous reference frame (SRF) for PLL control scheme is illustrated as Fig. 1, where $k_{p, i}$ and $k_{i, i}$ are Proportional-Integrational (PI) parameters of the $i^{\text {th }}$ PLL; $x_{1, i}$ is the output state variable of the $i^{\text {th }}$ PLL integral controller; $s_{p l l, i}$ represents the phase angle of the $i^{\text {th }}$ converter's terminal voltage measured by its PLL. Thus, the reduced-order model of the $i^{\text {th }}$ converter in Fig. 1 can be rewritten by:

$$
\left\{\begin{array}{l}
\frac{d}{d t} \Delta \boldsymbol{X}_{r, i}=\boldsymbol{A}_{r, i} \Delta \boldsymbol{X}_{r, i}+\boldsymbol{B}_{r, i} \Delta \boldsymbol{U}_{i} \\
\Delta \boldsymbol{I}_{i}=\boldsymbol{C}_{r, i} \Delta \boldsymbol{X}_{r, i}
\end{array} i=1, \cdots, n\right.
$$


where

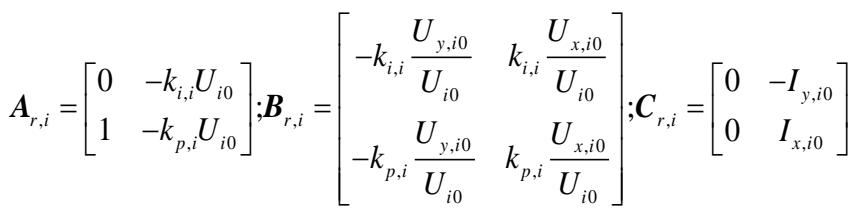

where $\boldsymbol{A}_{r, i}, \boldsymbol{B}_{r, i}$ and $\boldsymbol{C}_{r, i}$ represent state matrix, control matrix, and output matrix corresponding to the reduced-order model; $\boldsymbol{X}_{r, i}=\left[x_{1, i}, \mathrm{~d}_{p l l, i}\right]^{T} ; U_{i}$ denotes the magnitude of terminal voltage of the $i^{\text {th }}$ converter; the subscript 0 denotes the steady state value of the variables. The detailed derivation of equation (3) is elaborated in Appendix A.

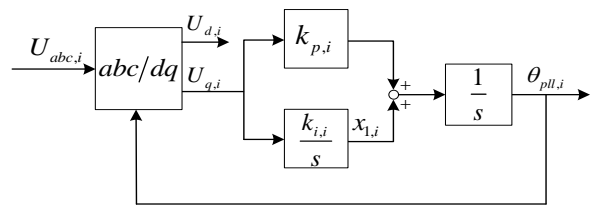

Fig. 1 The linearized model of an SRF-PLL

By combining (2) and (3), the small-signal model of the HMCS-DPLL including both the reduced-order converters and power network can be represented by:

$$
\begin{gathered}
\frac{d}{d t} \Delta \boldsymbol{X}_{r}=\boldsymbol{A}_{h e_{-} s y s} \Delta \boldsymbol{X}_{r} \\
\boldsymbol{A}_{h e_{-} s y s}=\operatorname{diag}\left(\boldsymbol{A}_{r, i}\right)+\operatorname{diag}\left(\boldsymbol{B}_{r, i}\right)(\boldsymbol{Z} \otimes \boldsymbol{\alpha}(s)) \operatorname{diag}\left(\boldsymbol{C}_{r, i}\right)
\end{gathered}
$$

where $\Delta \boldsymbol{X}_{r}=\left[\Delta \boldsymbol{X}_{r, 1}{ }^{\mathrm{T}}, \ldots, \Delta \boldsymbol{X}_{r, n}{ }^{\mathrm{T}}\right] ; \operatorname{diag}(\epsilon)$ represents a diagonal matrix; $\boldsymbol{A}_{\text {he_sys }}$ represents the state matrix of the HMCS-DPLL.

Based on (4), the characteristic equation of the reduced-order HMCS-DPLL can be represented by:

$$
\operatorname{det}\left[\lambda \boldsymbol{I}-\boldsymbol{A}_{h e_{-} s y s}\right]=0
$$

The characteristic equation (6) can be used for the small-signal stability analysis of the HMCS-DPLL. The system is stable if and only if all the eigenvalues in the characteristic equation (6) are in the left-half of the complex plane.

\section{SMALL-SIGNAL StABILITY BOUNDARY OF HMCS-DPLL}

It is challenging to directly use the characteristic equation (6) to derive the small-signal stability boundary of the HMCSDPLL due to the complex interaction between the power network and interconnected converters with multiple PLLs. Especially, this derivation complexity is further increased when the PI control parameters of multiple PLLs are different in a HMCS-DPLL. To address the challenges, we first analyze the stability of a homogeneous system dominated by the dynamics of the PLLs with identical PI control parameters. Then, the analysis results will be extended to the HMCS-DPLL for facilitating its small-signal stability analysis. On this basis, we further derive the stability boundary condition and discuss the instability mechanism insights in the HMCS-DPLL.

\section{A. Small-signal stability analysis of a homogeneous system dominated by PLLs}

For a homogeneous system dominated by the PLLs, the statespace model of each converter satisfies $\boldsymbol{A}_{r, i}=\boldsymbol{A}, \boldsymbol{B}_{r, i}=\boldsymbol{B}$, and $\boldsymbol{C}_{r, i}=\boldsymbol{C}, i=1, \ldots, n$. Under this condition, small-signal stability in such a system can be analyzed by the following characteristic equation.

$$
\begin{aligned}
& \operatorname{det}\left[\lambda \boldsymbol{I}_{n}-\boldsymbol{A}_{h o_{-} s y s}\right] \\
& =\operatorname{det}\left[\lambda \boldsymbol{I}_{n}-\left(\left(\boldsymbol{I}_{n} \otimes \boldsymbol{A}\right)+\left(\boldsymbol{I}_{n} \otimes \boldsymbol{B}\right)(\boldsymbol{Z} \otimes \alpha(s))\left(\boldsymbol{I}_{n} \otimes \boldsymbol{C}\right)\right)\right]=0
\end{aligned}
$$

where $\boldsymbol{I}_{n}$ is a $n$-dimension identity matrix; $\boldsymbol{A}_{h o_{-} s y s}$ represents the state matrix of the homogeneous multi-converter system.

To simplify the small-signal stability analysis in such a system, it is noticed that matrix $\boldsymbol{Z}$ is a positive definite and can be diagonalized with all positive eigenvalues [18]. Based on modal decoupling theory, there is a matrix $\boldsymbol{W}$ that can decompose matrix $\boldsymbol{Z}$ into a diagonal matrix in which the diagonal elements consist of the eigenvalues $\left(\epsilon_{i}, i=1, \ldots, n\right)$ in the order of $\epsilon_{1} \geq \zeta \geq \sigma_{i} \geq \mid \geq \epsilon_{n} \geq 0$. That is,

$$
\boldsymbol{W}^{-1} \boldsymbol{Z W}=\mathbf{S}=\operatorname{diag}\left(\sigma_{i}\right) \quad i=1, \ldots, n
$$

Combining (7) with (8) yields,

$$
\begin{aligned}
& \operatorname{det}\left[\lambda \boldsymbol{I}_{n}-\boldsymbol{A}_{h o_{-s y s}}^{e q}\right] \\
& =\operatorname{det}\left[\lambda \boldsymbol{I}_{n}-\left(\left(\boldsymbol{I}_{n} \otimes \boldsymbol{A}\right)+\left(\boldsymbol{I}_{n} \otimes \boldsymbol{B}\right)(\mathbf{S} \otimes \alpha(s))\left(\boldsymbol{I}_{n} \otimes \boldsymbol{C}\right)\right)\right]=0
\end{aligned}
$$

Based on (9), a homogeneous $n$-converter system in the physical-coordinate can be decoupled into $n$ subsystems in the modal-coordinate, and each subsystem has a single converter connected to the infinite node via an equivalent line reactance $\epsilon_{i}(i=1, \ldots, n)$, which can be characterized by (10) below. Thus, the homogeneous system is stable if and only if all the subsystems in (10) are stable.

$$
\begin{aligned}
& \operatorname{det}\left[\lambda \boldsymbol{I}-\boldsymbol{A}_{h o_{-} s y s, i}^{\text {eq }}\right] \\
& =\operatorname{det}\left[\lambda \boldsymbol{I}-\left(\boldsymbol{A}+\boldsymbol{B}\left(\sigma_{i} \cdot \alpha(s)\right) \boldsymbol{C}\right)\right]=0 \quad i=1, \ldots, n
\end{aligned}
$$

Furthermore, the small-signal stability of the homogeneous system can be characterized by the most critical decoupled subsystem. Since each converter has the same control configuration and parameters in the homogeneous system, the stability of each subsystem depends on $\epsilon_{i}(i=1, \ldots, n)$ when the control configuration and parameters are given. The large $\epsilon_{\mathrm{i}}$ means its corresponding subsystem is more likely to be unstable. In other words, the small-signal stability of the entire homogeneous system depends on the most critical decoupled subsystem which has the largest eigenvalue $\epsilon_{1}$ of matrix $\boldsymbol{Z}$ as the equivalent line reactance connected to the grid. Hence, the small-signal stability of the entire homogeneous system can be analyzed based on the characteristic equation of the most critical decoupled subsystem:

$$
\operatorname{det}\left[\lambda \boldsymbol{I}-\boldsymbol{A}_{h o_{-} s y s, 1}^{e q}\right]=\operatorname{det}\left[\lambda \boldsymbol{I}-\left(\boldsymbol{A}+\boldsymbol{B}\left(\sigma_{1} \cdot \alpha(s)\right) \boldsymbol{C}\right)\right]=0
$$

\section{B. Small-signal Stability Analysis of a HMCS-DPLL}

For a HMCS-DPLL, each converter may have different PI control parameters for their PLLs. That is, $\boldsymbol{A}_{r_{z}} \boldsymbol{A}_{r, j}, \boldsymbol{B}_{r_{z}, j} \boldsymbol{B}_{r, j}$, and $\boldsymbol{C}_{r, j} \boldsymbol{C}_{r, j, j}, j$, and $i, j=1, \ldots, n$. In such a system, the smallsignal stability can be analyzed by the characteristic equation (6). Due to the nature of the heterogeneous system, such a characteristic equation cannot be directly decoupled. To simplify the small-signal stability analysis, we define an equivalent multi-infeed homogeneous system, where each equivalent single-converter has the same state matrix $\overline{\boldsymbol{A}}$, control matrix $\overline{\boldsymbol{B}}$, and output matrix $\overline{\boldsymbol{C}}$, which can be represented by: 


$$
\overline{\boldsymbol{A}}=\sum_{i=1}^{n} p_{i} \boldsymbol{A}_{r, i} ; \quad \overline{\boldsymbol{B}}=\sum_{i=1}^{n} p_{i} \boldsymbol{B}_{r, i} ; \quad \overline{\boldsymbol{C}}=\sum_{i=1}^{n} p_{i} \boldsymbol{C}_{r, i}
$$

where $p_{i}=u_{i 1} v_{i 1}(i=1, \ldots, n)$ is the participation factor and it satisfies $\sum_{i=1}^{n} p_{i}=1, p_{i}>0 ; u_{i 1}$ and $v_{i 1}$ are the $i^{\text {th }}$ elements of normalized left and right eigenvectors $\boldsymbol{u}_{1}{ }^{T}$ and $\boldsymbol{v}_{1}$ for the largest eigenvalue ${ }_{1}$ of Kron-reduced reactance matrix $\boldsymbol{Z}$, respectively. It is worth noting that $p_{i}$ can reflect the impact degree of the $i^{\text {th }}$ converter parameters on the dominant modes of the HMCSDPPL [18]. That is, $p_{i}$ can further characterize the impact of the PLL of $i^{\text {th }}$ converter on the small-signal stability of HMCSDPLL.

Thus, the state matrix $\bar{A}_{h o_{-} s y s}$ of the equivalent homogeneous system can be represented as:

$$
\overline{\boldsymbol{A}}_{h o_{-} s y s}=\left(\boldsymbol{I}_{n} \otimes \overline{\boldsymbol{A}}\right)+\left(\boldsymbol{I}_{n} \otimes \overline{\boldsymbol{B}}\right)(\boldsymbol{Z} \otimes \alpha(s))\left(\boldsymbol{I}_{n} \otimes \overline{\boldsymbol{C}}\right)
$$

The following Lemma 1 proves that the small-signal dynamics of the HMCS-DPLL can be characterized by the equivalent homogeneous system.

Lemma 1. Define $\boldsymbol{\gamma}$ and $\bar{\lambda}$ are the dominant eigenvalue function of the HMCS-DPLL and its equivalent homogeneous system, respectively, which are pertinent to the system's dominant eigenvalues. Then, the loci of $x_{\text {and }} \bar{\lambda}$ have the following relationship:

$$
\lambda=\bar{\lambda}+\boldsymbol{O}\left(\left\|\boldsymbol{A}_{h e_{-} s y s}-\overline{\boldsymbol{A}}_{h o_{-} s y s}\right\|\right)
$$

where $\boldsymbol{O}(\epsilon)$ is the second-order and much higher order approximate error of a function.

Proof: Based on the small-signal stability analysis of the homogeneous system in Section III.A, it is known that the stability of the equivalent homogeneous system depends on its most critical decoupled subsystem. Thus, $\bar{\lambda}$ is also the dominant eigenvalue function of the most critical decoupled subsystem's state matrix $\overline{\boldsymbol{A}}_{h o}^{e q}{ }_{\text {ss. }}$, which is corresponding to the largest eigenvalue $\epsilon_{1}$ of matrix $\boldsymbol{Z}$. Also, we define $\boldsymbol{\mu}^{T}$ and $\boldsymbol{v}$ as the normalized left and right eigenvectors of $\overline{\boldsymbol{A}}_{h o_{-} y s, 1}^{\text {eq }}$ respectively. That is,

$$
\bar{\lambda}=\boldsymbol{\mu}^{T} \overline{\boldsymbol{A}}_{h o_{-} s y, 1}^{e q} \boldsymbol{\nu}=\boldsymbol{\mu}^{T} \overline{\boldsymbol{A}} \boldsymbol{\nu}+\boldsymbol{\mu}^{T} \overline{\boldsymbol{B}}\left(\sigma_{1} \cdot \alpha(s)\right) \overline{\boldsymbol{C}} \boldsymbol{v}
$$

Then, the normalized left and right eigenvector of $\overline{\boldsymbol{A}}_{h 0_{-s y s}}$ for the dominant eigenvalue function $\bar{\lambda}$ can be further expressed as $\boldsymbol{u}_{1}^{T} \otimes \boldsymbol{\mu}^{T}$ and $\boldsymbol{v}_{1} \otimes \boldsymbol{v}$. That is

$$
\begin{gathered}
\bar{\lambda}=\left[\boldsymbol{u}_{1}^{T} \otimes \boldsymbol{\mu}^{T}\right] \overline{\boldsymbol{A}}_{h o_{-S y s}}\left[\boldsymbol{v}_{1} \otimes \boldsymbol{v}\right] \\
1=\left[\boldsymbol{u}_{1}^{T} \otimes \boldsymbol{\mu}^{T}\right]\left[\boldsymbol{v}_{1} \otimes \boldsymbol{v}\right]
\end{gathered}
$$

By the matrix perturbation theory (Theorem 2.3 in [21]), we consider $\boldsymbol{A}_{h e_{-} s y s}$ as a perturbation of $\overline{\boldsymbol{A}}_{h o_{-} s y s}$. Then, the loci of the dominant eigenvalue function วfor $\boldsymbol{A}_{\text {he_sys }}$ is given as:

$$
\begin{aligned}
\lambda= & {\left[\boldsymbol{u}_{1}^{T} \otimes \boldsymbol{\mu}^{T}\right] \boldsymbol{A}_{h e_{-} s y}\left[\boldsymbol{v}_{1} \otimes \boldsymbol{v}\right]+\boldsymbol{O}\left(\left\|\boldsymbol{A}_{h e_{-} s y s}-\overline{\boldsymbol{A}}_{h o_{-} s y s}\right\|\right) } \\
= & {\left[\boldsymbol{u}_{1}^{T} \otimes \boldsymbol{\mu}^{T}\right]\left[\operatorname{diag}\left(\boldsymbol{A}_{r, i}\right)+\operatorname{diag}\left(\boldsymbol{B}_{r, i}\right)(\boldsymbol{Z} \otimes \boldsymbol{\alpha}(s)) \operatorname{diag}\left(S_{B i} \cdot \boldsymbol{C}_{r, i}\right)\right] } \\
& \cdot\left[\boldsymbol{v}_{1} \otimes \boldsymbol{v}\right]+\boldsymbol{O}\left(\left\|\boldsymbol{A}_{h e_{-} s y s}-\overline{\boldsymbol{A}}_{h o_{-} s y s}\right\|\right) \\
= & \boldsymbol{\mu}^{T} \overline{\boldsymbol{A}} \boldsymbol{\nu}+\boldsymbol{\mu}^{T} \overline{\boldsymbol{B}}\left(\sigma_{1} \cdot \alpha(s)\right) \overline{\boldsymbol{C}} \boldsymbol{v}+\boldsymbol{O}\left(\left\|\boldsymbol{A}_{h e_{-} s y s}-\overline{\boldsymbol{A}}_{h o_{-} s y s}\right\|\right) \\
= & \bar{\lambda}+\boldsymbol{O}\left(\left\|\boldsymbol{A}_{h e_{-s y s}}-\overline{\boldsymbol{A}}_{h o_{-} s y s}\right\|\right)
\end{aligned}
$$

This concludes the proof, and the detailed derivation for (18) is presented in Appendix B.

Based on Lemma 1, the small-signal dynamics of the HMCSDPLL can be approximated by the equivalent homogeneous system. According to the analysis in Section III. A, it is known that the equivalent homogeneous multi-converter system can be decoupled by $n$ subsystems for the small-signal stability analysis, and the small-signal stability of the equivalent homogeneous system can be analyzed based on the characteristic equation of the most critical decoupled subsystem:

$$
\operatorname{det}\left[\bar{\lambda} \boldsymbol{I}-\overline{\boldsymbol{A}}_{h o_{-} s y s, 1}^{e q}\right]=\operatorname{det}\left[\bar{\lambda} \boldsymbol{I}-\left(\overline{\boldsymbol{A}}+\overline{\boldsymbol{B}}\left(\sigma_{1} \cdot \alpha(s)\right) \overline{\boldsymbol{C}}\right)\right]=0
$$

\section{Stability boundary condition of HMCS-DPLL}

Based on (19) and Routh-Hurwitz stability criterion [22], the stability boundary condition of the HMCS-DPLL can be derived in terms of the ratio of the weighted PI gains of PLLs $K_{\mathrm{I}_{-} \Sigma} / K_{\mathrm{P}_{-} \Sigma}$ :

$$
\frac{K_{I_{-} \Sigma}}{K_{P_{-} \Sigma}} \leq K_{c}=\frac{U_{0}^{2} \omega_{0}}{\sigma_{1} P_{0}}-\frac{Q_{0} \omega_{0}}{P_{0}}-\tau
$$

where $K_{P_{-} \Sigma}=\sum_{i=1}^{n} p_{i} k_{p, i}$ and $K_{I_{-} \Sigma}=\sum_{i=1}^{n} p_{i} k_{i, i}$ represent the weighted PI control parameters of all PLLs through the coefficient $p_{i} ; U_{0}$, $P_{0}$, and $Q_{0}$ denote the steady-state terminal voltage, active power, and reactive power of each converter, which can be considered as the identical value among converters at the planning stage. The detailed derivation of (20) can be founded in Appendix C.

According to the stability boundary condition (20), the stability of the HMCS-DPLL can be easily identified without the need for modal analysis based on the full-order model. When the ratio of the weighted PI gains $K_{\mathrm{I}_{-} \Sigma} / K_{\mathrm{P}_{-} \Sigma}$ of all PLLs is smaller than or equal to the stability boundary value $K_{c}$ (i.e., $K_{\mathrm{I}_{-} \Sigma} / K_{\mathrm{P}_{-} \Sigma} \leqslant K_{\mathrm{c}}$ ), the PI parameter setting of all PLLs are proper and the stability of the HMCS-DPLL is thus guaranteed. Otherwise, the PI parameter setting of all PLL are not proper, which may cause the instabitiy risk of the HMCS-DPLL. Thus, the stability margin of the HMCS-DPLL can be calculated by the difference between the stability boundary value $K_{c}$ and the ratio of the weighted PI gains $K_{\mathrm{I}_{-} \Sigma} / K_{\mathrm{P}_{-} \Sigma}$ of all PLLs (i.e., $K_{\mathrm{c}-}$ $K_{\mathrm{I}_{-} \Sigma} / K_{\mathrm{P}_{-} \Sigma}$ ). Table I below summarizes the main steps of using the boundary condition (20) to identify the rationality of PLLs' control parameters and the stabitiy margin of the HMCS-DPLL.

Table I The stability analysis of the HMCS-DPLL based on (20).

Input: Kron-reduced reactance matrix $\boldsymbol{Z}$;

Different PLL PI control parameters $k_{p, i}$ and $k_{i, i}(i=1, \ldots, n)$; Steady-state values $P_{0}, Q_{0}, U_{0}, \tau$.

\section{Steps:}

1) Calculate $\epsilon_{1}$ and $p_{i}(i=1, \ldots, n)$;

2) Calculate $K_{I_{-} \Sigma} / K_{P_{-} \Sigma}$;

3) Calculate the critical value $K_{c}$ based on (20).

\section{Output:}

If $K_{I_{-} \Sigma} / K_{P_{-} \Sigma} \leq K_{c}$, the parameter design for PLLs is proper;

If $K_{I_{-} \Sigma} / K_{P_{-} \Sigma}>K_{c}$, the parameter design for PLLs is improper; 
Then, the stability margin of the HMCS-DPLL under the designed control parameters can be characterized by the value of $K_{c}-K_{I_{-} \Sigma} / K_{P_{-} \Sigma}$.

Also, the stability boundary condition (20) allows us to explore the instability mechanism of the HMCS-DPPL by analyzing how the interaction among different PLL PI control parameters under different network structure and operating conditions affects the stability of the HMCS-DPLL.

(1) Impact of network structure and converter operating conditions

As shown in (20), the stability of the HMCS-DPPL depends on network structure parameters (i.e., $\epsilon_{1}$ ) and the operating conditions of converters (i.e., $P_{0}$ ) when the PLL control parameters are given. $\boldsymbol{~}_{1}$ is the largest eigenvalue of matrix $\boldsymbol{Z}$ representing the power network interconnecting PLL-based converters. The stability boundary value $K_{c}$ changes with network topology and parameters and thus affect the stability of the HMCS-DPPL. When $\sigma_{1}$ increases (i.e., the power network becomes weak), $K_{c}$ decreases and thus reduces the small-signal stability margin (i.e., $K_{\mathrm{c}}-K_{\mathrm{I}_{-} \Sigma} / K_{\mathrm{P}_{-} \Sigma}$ ) of the HMCS-DPPL or even becomes unstable. Hence, the control parameters of PLLs should be designed to match the network structure. Also, the stability boundary value $K_{c}$ changes with the active power injection $P_{0}$ of each converter. When the injected active power $P_{0}$ increases, the decrease in $K_{c}$ reduces the small-signal stability margin (i.e., $K_{\mathrm{c}}-K_{\mathrm{I}_{-} \Sigma} / K_{\mathrm{P}_{-} \Sigma}$ ) of the HMCS-DPPL, which increases the instability risk of the HMCS-DPLL.

\section{(2) Impact of PLL control parameters}

Equation (20) shows the stability of the HMCS-DPPL also depends on the ratio $K_{\mathrm{I}_{\Sigma} \Sigma} / K_{\mathrm{P}_{-} \Sigma}$ of the weighted PI gains of all coupling PLLs when network structure parameters and the operating conditions of converters are given. The ratio $K_{\mathrm{I}_{\Sigma}} / K_{\mathrm{P}_{\Sigma} \Sigma}$ is mainly related to two factors: PI parameters of all PLLs (i.e., $k_{p, i}, k_{i, i}(i=1, \ldots, n)$ ), and the participation factor (i.e., $\left.p_{i}(i=1, \ldots, n)\right)$ which can be calculated from matrix $\boldsymbol{Z}$.

Specifically, $p_{i}$ determines the impact degree of the PLL dynamics of the $i^{\text {th }}$ converter on the stability of the HMCSDPLL. A larger value of $p_{i}$ means that the PLL PI control parameters of $i^{\text {th }}$ converter have a greater impact on the ratio $K_{\mathrm{I}_{-}}$ ${ }_{\Sigma} / K_{\mathrm{P}_{-} \Sigma}$ and thus on the small-signal stability of the HMCSDPPL. Further, multiple PLLs constitute an equivalent singleconverter system through $p_{i}(i=1, \ldots, n)$ whose equivalent PLL PI control parameters are $K_{\mathrm{P}_{-} \Sigma}, K_{\mathrm{I}_{-} \Sigma}$. Based on lemma 1 , the stability of the HMCS-DPPL is determined by the dynamics of the equivalent single-converter $(\bar{A}, \bar{B}, \bar{C})$ consisting of all PLLs coupled dynamically through the power network, instead of just the dynamics of certain single PLL. In other words, the different PLL dynamics collectively affect the stability of the HMCSDPLL, which can be characterized by the weighted PI control parameters $\left(K_{\mathrm{P}_{-} \Sigma}, K_{\mathrm{I}_{-} \Sigma}\right)$.

Particularly, the stability of the HMCS-DPPL is bounded by the upper and lower limits of ratio $k_{i, i} / k_{p, i}(i=1, \ldots, n)$. Based on a basic property of the fractional inequalities, the ratio $K_{\mathrm{I}_{-} \Sigma} / K_{\mathrm{P}_{-} \Sigma}$ meets the following inequality:

$$
\left(\frac{k_{i, i}}{k_{p, i}}\right)_{\min } \leq \frac{K_{I_{-} \Sigma}}{K_{P_{-} \Sigma}}=\frac{\sum_{i=1}^{n} p_{i} k_{i, i}}{\sum_{i=1}^{n} p_{i} k_{p, i}} \leq\left(\frac{k_{i, i}}{k_{p, i}}\right)_{\text {max }}
$$

where $\left(k_{i, i} / k_{p, i}\right)_{\min }$ and $\left(k_{i, i} / k_{p, i}\right)_{\max }$ represent the minimum value and the maximal value of ratio $k_{i, i} / k_{p, i}, i=1, \ldots, n$.

According to (21), it is known that the ratio $K_{\mathrm{I}_{-} \Sigma} / K_{\mathrm{P}_{-} \Sigma}$ is bounded by its upper limit $\left(k_{i, i} / k_{p, i}\right)_{\max }$ and lower limit $\left(k_{i, i} / k_{p, i}\right)_{\min }$. Thus, for the given stability boundary value $K_{c}$, the small-signal stability of the HMCS-DPLL is bounded by its upper limit $\left(k_{i, i} / k_{p, i}\right)_{\max }$ and lower limit $\left(k_{i, i} / k_{p, i}\right)_{\min }$. When the interaction among different PLLs through the ac network is ignored, $\left(k_{i, i} / k_{p, i}\right)_{\max }$ can be used to approximate $K_{\mathrm{I}_{-} \Sigma} / K_{\mathrm{P}_{-} \Sigma}$, but the stability anlaysis and stability margin evaluation based on $K_{\mathrm{c}}-$ $\left(k_{i, i} / k_{p, i}\right)_{\max }$ may give a relatively conservative or even incorrect results. This conclusion will be demonstrated in the next section.

\section{CAse STUdies}

In this section, the analytical results and proposed method for the stability evaluation of the HMCS-DPLL are validated by the modal analysis and the electromagnetic transient time-domain simulation on a 9-converter heterogeneous system as shown in Fig. 2. In this system, 9 converters are interconnected via a 39bus meshed network, which is the same as the one in the standard IEEE 39-bus system in [23] and the line parameters are modified by setting Üas 0.1 . The control parameters of converters are illustrated in Table II. Converters 1 6 use DC voltage outer-loop control mode, and converters 7 9 adopt power outer-loop control mode. All the converters in the system are equipped with the SRF-PLLs. To verify the analytical results and the proposed method derived in the previous section, three cases are considered for the impact investigation on the stability of a 9-converter heterogeneous system due to 1) the PLL control parameters, 2) network parameters, and 3) converter operating conditions.

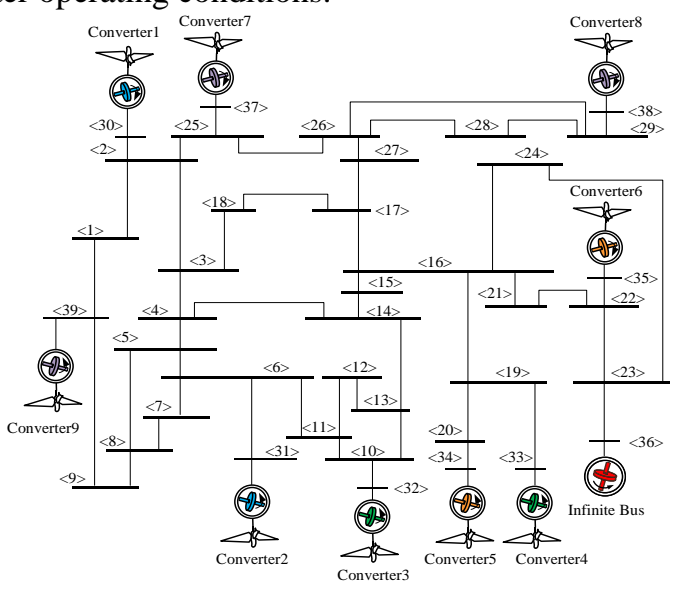

Fig. 2 The 9-converter heterogeneous system

Table II Parameters of converters

\begin{tabular}{ll}
\hline Filter inductance, filter capacitance, dc capacitance & $0.05,0.05,0.038$ p.u. \\
PI parameters of current control loop & 1,10 \\
PI parameters of the active power control loop & $0.5,5$ \\
PI parameters of the DC voltage control loop & $0.5,5$ \\
Parameters of the voltage feedforward filter & 0.001 \\
PI parameters of the PLL & 20,9100 \\
The steady-state value of $P_{0}, Q_{0}$ of each converter & 1.0 p.u., 0 p.u.
\end{tabular}




\section{1) Case 1: Impact of PLL control parameters}

In this case, the stability boundary condition in (20) is verified on a 9-converter heterogeneous system with the given network parameters and converter power injection. The network is the original power network used in the IEEE 39-bus system. The power injection of each converter is set as 1.0 p.u.. The $\epsilon_{1}$ and stability boundary value $K_{c}$ in (20) are calculated, and they are $\epsilon_{1}=0.5032$ and $K_{c}=624.16$. Also, we consider three groups of different PLL PI control parameters (1) (3) for all converters in the 9-converter heterogeneous system. The PI control parameters are presented in Table III. The resulting ratios of the PI weighted gains $K_{\mathrm{I}_{-} \Sigma} / K_{\mathrm{P}_{-} \Sigma}$ in (20) are presented in Table IV. Also, Table IV compares the dominant eigenvalues of the system evalauted based on the full-order model in (1) and (2) and the reduced-order model in (19) under three groups of different ratios $K_{\mathrm{I}_{\perp} \Sigma} / K_{\mathrm{P}_{-} \Sigma}$. In addtion, a disturbance is applied to this system under each group of PLLs' PI control parameters and the given network parameters and converter power injection, in order for the electromagnetic time-domain simulation using MATLAB/Simulink. The disturbance is applied to the infinite bus (i.e. node 36 in the Fig. 2) in the system at 0.10 s to cause voltage rise by $10 \%$ and then is cleared at $0.12 \mathrm{~s}$. Fig. 3 shows the active power trajectories of all converters in the 9-converter heterogeneous system following the disturbance for three groups of different ratios $K_{\mathrm{I}_{-} \Sigma} / K_{\mathrm{P}_{-} \Sigma}$ shown in Table IV.

Table III Three groups of PLLs' PI control Parameters

\begin{tabular}{ccccc}
\hline Group & Converter 1 2 & Converter 3 4 & Converter 5 6 & Converter 7 9 \\
(1) & 20,9100 & 15,8750 & 10,8450 & 15,8250 \\
\hline Group & Converter 1 2 & Converter 3 4 & Converter 5 6 & Converter 7 9 \\
(2) & 20,9500 & 14,9300 & 6,8700 & 15,9200 \\
\hline Group & Converter 1 2 & Converter 3 4 & Converter 5 6 & Converter 7 9 \\
(3) & 18,9500 & 14,9300 & 7,8700 & 11,9200 \\
\hline
\end{tabular}

It can be observed from Table IV and Fig. 3 that the stability boundary condition in (20) can effectively identify the stability and stability margin of the 9-converter heterogeneous system. As shown in Table IV, when $K_{\mathrm{I}_{-} \Sigma} / K_{\mathrm{P}_{-} \Sigma}=551.69$ is smaller than $K_{c}=624.16$, the dominant eigenvalues of the system full-order model are in the left-half of the complex plane, which indicates that the system is stable with a certain stability and PLL PI control parameters are designed appropriately margin; when $K_{\mathrm{I}_{-}}$ ${ }_{\Sigma} / K_{\mathrm{P}_{-} \Sigma}=622.38$ is almost equal to $K_{c}=624.16$, the dominant eigenvalues of the system full-order model are almost at the imaginary axis, which indicates the system is critically stable; when $K_{\mathrm{I}_{-} \Sigma} / K_{\mathrm{P}_{-} \Sigma}=717.21$ is larger than $K_{c}=624.16$, the dominant eigenvalues of the system full-order model are in the right-half of the complex plane, which suggests the system is unstable and PLL PI control parameters are designed inappropriately. The similar observations can also be obtained from Fig. 3 based on the results of electromagnetic transient simulation: when $K_{\mathrm{I}_{-} \Sigma}$ $/ K_{\mathrm{P}_{-} \Sigma}=551.69<K_{c}=624.16$, the convergence oscillation can be seen, which means the system is stable with certain margin and PLL PI control parameters are designed properly; when $K_{\mathrm{I}_{-} \Sigma}$ $/ K_{\mathrm{P}_{-} \Sigma}=622.38$ is almost equal to $K_{c}=624.16$, the undamped oscillation can be seen, which suggests the system is critically stable; when $K_{\mathrm{I}_{-} \Sigma} / K_{\mathrm{P}_{-} \Sigma}=717.21>K_{c}=624.16$, the divergent oscillation can be seen, which indicates the system is unstable and PLL PI control parameters are not designed properly. The consistent observations between the modal analysis and electromagnetic transient simulation verifies the effectiveness of the stability boundary condition in (20) for the small-signal stability assessment and PLL PI control design in the HMCSDPLL.

Table IV The dominant eigenvalues of the 9-conveter heterogeneous system evaluated based on the full-order model and the reduced-order model under three groups PLL PI control parameters (1)-(3).

\begin{tabular}{cccc}
\hline No. & $K_{\mathrm{I}_{-} \Sigma} / K_{\mathrm{P}_{-} \Sigma}$ & $\begin{array}{c}\text { Full-order } \\
\text { model }\end{array}$ & $\begin{array}{c}\text { Reduced-order } \\
\text { HMCS-DPLL model }\end{array}$ \\
\hline (1) & 551.69 & $-1.3475 \pm 94.8595 \mathrm{i}$ & $-0.9280 \pm 93.9045 \mathrm{i}$ \\
$(2)$ & 622.38 & $-0.2709 \pm 97.1733 \mathrm{i}$ & $-0.0217 \pm 97.2275 \mathrm{i}$ \\
(3) & 717.21 & $0.3639 \pm 97.4314 \mathrm{i}$ & $0.9793 \pm 97.0665 \mathrm{i}$ \\
\hline
\end{tabular}
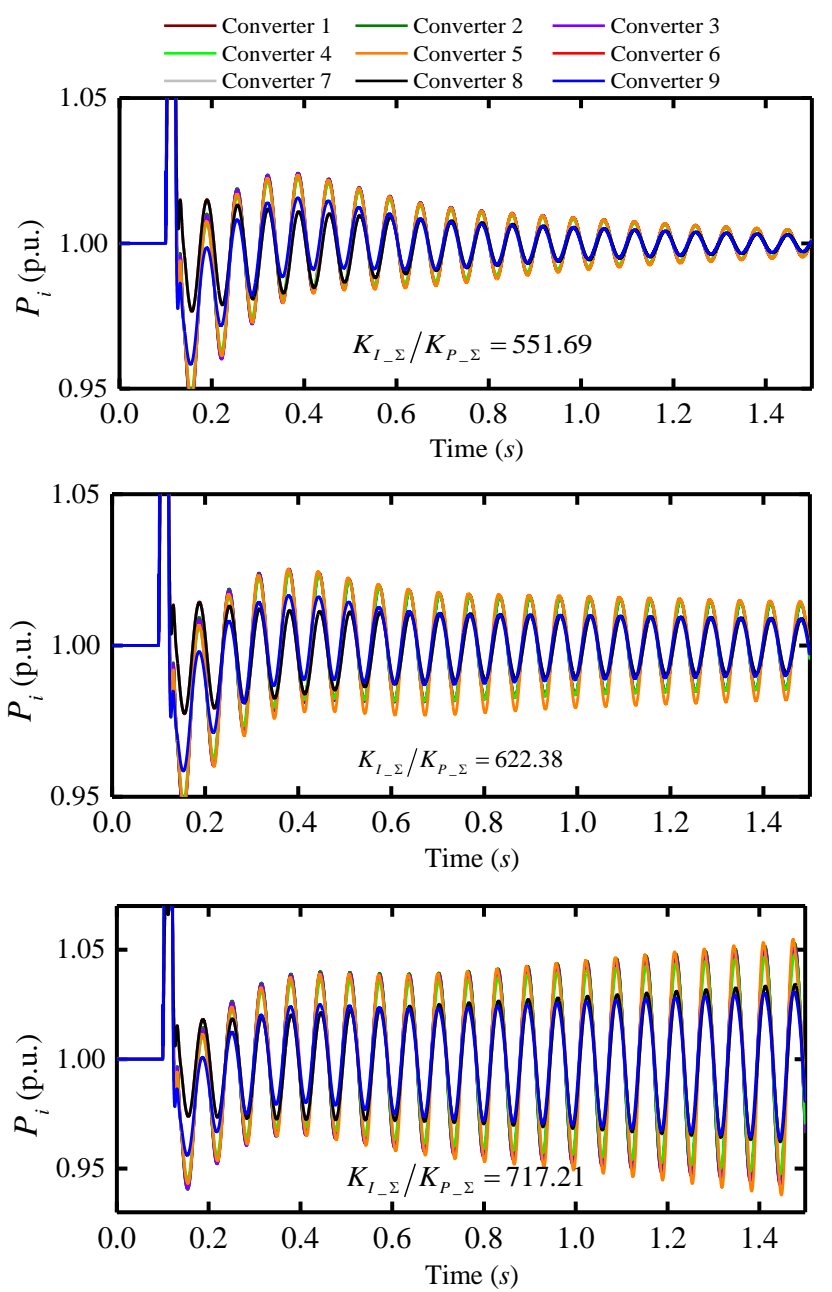

Fig. 3 The active power trajectories of converters in the 9-converter heterogeneous system under three groups PLL PI control parameters following a disturbance.

Also, it can be observed from Table IV that the derived reduced-order model in (19) can effectively approximate the full-order model in (1) and (2) for the small-signal stability of the HMCS. In Table IV, the $3^{\text {rd }}$ and $4^{\text {th }}$ columns are the dominant eignevalues of the system evaluated based on the fullorder model in (1) and (2) and the reduced-order model under three groups of different ratios $K_{\mathrm{I}_{\Sigma} \Sigma} / K_{\mathrm{P}_{-} \Sigma}$. Their relative errors are $1.099 \%, 0.262 \%$, and $0.734 \%$, respectively, which demonstrates the effectiveness of using the most critical decoupled subsystem to evaluate the small-signal stability of 
the full-order HMCS. Since the state matrix $\bar{A}_{h o_{-5 s}, 1}^{e q}$ in (19) is only a 2-order matrix, the derived reduced-order model greatly reduces the computational burden and thus improves the applicability of modal analysis in a practical HMCS-DPLL.

Furthermore, we verify that our analytical results of the impact of different PLL PI control dynamics on the stability of the HMCS-DPLL. To this end, we evaluate the participation factors of different PLL PI control parameters and the ratio $k_{i, i} / k_{p, i}$ of PLL PI control parameters for each converter in the 9converter heterogeneous system under three groups of different PLL PI control parameters. The results are shown in Table V and, respectively.

Table V Participation factors of all converter in the 9-converter system with the given network parameters.

\begin{tabular}{cccccc}
\hline Converter $i$ & 1 & 2 & 3 & 4 & 5 \\
$p_{i}$ & 0.1269 & 0.1270 & 0.1214 & 0.0908 & 0.0978 \\
\hline Converter $i$ & 6 & 7 & 8 & 9 & \\
$p_{i}$ & 0.0387 & 0.1313 & 0.1329 & 0.1332 & \\
\hline
\end{tabular}

It can be observed from Table $\mathrm{V}$ that the participation factors can quantify the impact of converter PLL control parameters on the stability of the HMCS-DPLL. In Table V, converters 7 9 have larger participation factors than the other converters, and the control parameters design for the PLLs of converters 7 9 is particularly crucial. Further, if the PLL PI control parameters of converters 7 9 are adjusted preferentially from " 11,9200 " to "20, 9000" under the scenario (3) in Fig. 3, the system will become stable following the disturbance, as illustrated in Fig. 4.

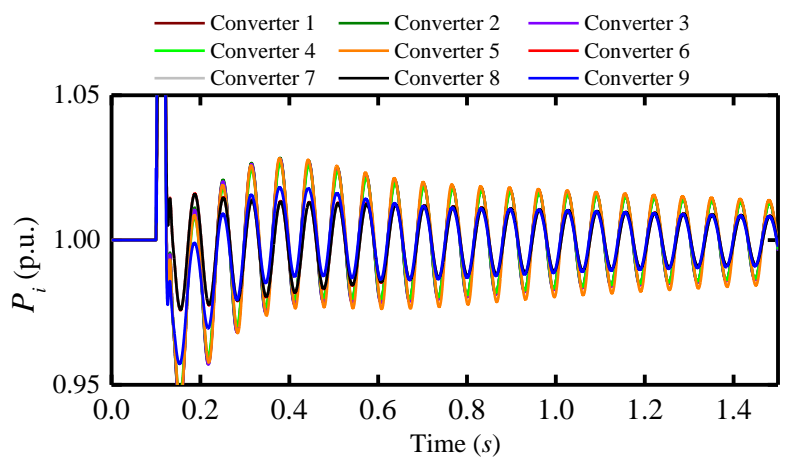

Fig. 4 The active power trajectories of converters in the 9-converter system under the PLL control parameters (3) following a disturbance when changing the PLLs' PI parameters of converter 7 9 from " 11,9200 " to " 20,9000 ".

Table VI The ratio $k_{i, i} / k_{p, i}$ of PLL control parameters under three groups PLL control parameters (1)-(3).

\begin{tabular}{cccccc}
\hline Group & Converter $i$ & $1 \sim 2$ & $3 \sim 4$ & $5 \sim 6$ & $7 \sim 9$ \\
(1) & $\left(k_{i, i} / k_{p, i}\right)_{\max }$ & 455 & 583.33 & $\mathbf{8 4 5}$ & 550 \\
\hline Group & Converter $i$ & $1 \sim 2$ & $3 \sim 4$ & $5 \sim 6$ & $7 \sim 9$ \\
(2) & $\left(k_{i, i} / k_{p, i}\right)_{\max }$ & 475 & 664.29 & $\mathbf{1 4 5 0}$ & 613.33 \\
\hline Group & Converter $i$ & $1 \sim 2$ & $3 \sim 4$ & $5 \sim 6$ & $7 \sim 9$ \\
(3) & $\left(k_{i, i} / k_{p, i}\right)_{\max }$ & 527.78 & 664.29 & $\mathbf{1 2 4 2 . 8 6}$ & 836.36 \\
\hline
\end{tabular}

The results of Table VI show when the upper limit $\left(k_{p, i} / k_{i, i}\right)_{\max }$ is used to approximate $K_{\mathrm{I}_{-} \Sigma} / K_{\mathrm{P}_{-} \Sigma}$ in (20), the incorrect analysis results may be obtained. In Table VI, the ratio $\left(k_{i, i} / k_{p, i}\right)_{\max }$ is 845 , 1450 , and 1242.86 under the above three groups of different PLL PI control parameters for all converters. If the upper limit $\left(k_{i, i} / k_{p, i}\right)_{\max }$ is used to approximate $K_{\mathrm{I}_{-} \Sigma} / K_{\mathrm{P}_{-} \Sigma}$ in (20), we will obtain the conclusions that the system is unstable under the above three groups PLL control parameters by comparing $\left(k_{i, i} / k_{p, i}\right)_{\max }$ with $K_{c}=624.16$. However, the system is stable under the groups of the PLL PI control parameters (1) and (2) when comparing $K_{\mathrm{I}_{-} \Sigma} / K_{\mathrm{P}_{-} \Sigma}$ with $K_{c}=624.16$. Thus, it may be not suitable to design the PLL control using the analysis results of approximaing $K_{\mathrm{I}_{-} \Sigma} / K_{\mathrm{P}_{-} \Sigma}$ in (20) by the upper limit $\left(k_{i, i} / k_{p, i}\right)_{\max }$.

\section{2) Case 2: Impact of network parameter}

In this case, the analytical results of the impact of network parameters on the stability of the HMCS-DPLL are verified on the 9-converter heterogeneous system with the given PLL control parameters and converter power injection. The PLL control parameters of each converter are set as the first group presented in Table III. Then, the network parameters are changed by coefficient $k$, which is proportional to the line length in the system. During the above process, the line parameter and network strength of the power network is changed, while the structure of the power network is not altered, which means the participation factors $p_{i}(i=1, \ldots, n)$ of all converters are the same as Table $\mathrm{V}$. Thus, the resulting $K_{\mathrm{I} \Sigma} / K_{\mathrm{P} \Sigma}$ in (20) is 551.69 . When $k$ is set as $0.80,1.12$, and 1.20 , accordingly, the values of the resulting $K_{c}$ are 780.23, 557.32, and 520.12. Table VII shows the values of $K_{c}$ and the dominant eignevalues of the system evalauted based on the full-order model in (1) and (2) and the reduced-order model in (19), when $k$ is set as $0.80,1.12$, and 1.20 . In addtion, a disturbance is applied to this system when $k$ is set as $0.80,1.12$, and 1.20 , respectively, for the electromagnetic time-domain simulation using MATLAB/Simulink. The disturbance is the same as the one used in case 1). Fig. 5 shows the active power trajectories of all converters in the 9-converter heterogeneous system following the disturbance when $k$ is set as $0.80,1.12$, and 1.20 .

Table VII The dominant eigenvalues of the 9-conveter system evaluated based on the full-order model and the reduced-order model when $k$ is set as $0.80,1.12$, and 1.20 .

\begin{tabular}{ccccc}
\hline$k$ & ढ $_{1}$ & $K_{c}$ & $\begin{array}{c}\text { Full-order } \\
\text { model }\end{array}$ & $\begin{array}{c}\text { Reduced-order } \\
\text { model }\end{array}$ \\
\hline 0.80 & 0.4026 & 780.23 & $-2.6655 \pm 94.8417 \mathrm{i}$ & $-2.3293 \pm 93.6421 \mathrm{i}$ \\
1.12 & 0.5636 & 557.32 & $-0.3601 \pm 94.4175 \mathrm{i}$ & $-0.0803 \pm 94.0528 \mathrm{i}$ \\
1.20 & 0.6039 & 520.12 & $0.4169 \pm 93.7864 \mathrm{i}$ & $0.4877 \pm 94.1478 \mathrm{i}$ \\
\hline
\end{tabular}

It can be observed from Table VII and Fig. 5 that the system loses its stability when the network becomes weak. In Table VII, when $k$ is increased from 0.80 to 1.20 , the $\epsilon_{1}$ is increased from 0.4026 to 0.6039 , which suggests the network becomes weak. As a result, $K_{c}$ is reduced from 780.23 to 520.13. Since $K_{\mathrm{I}_{\Sigma}} / K_{\mathrm{P}_{\Sigma} \Sigma}$ in (20) is 551.69 , the system loses its stability the stability boundary condition in (20) when $k$ is increased from 0.80 to 1.20 . Similarly, when $k$ is increased from 0.80 to 1.20 , the dominant eigenvalues evaluated based on the full-order model and the reduced-order model are also moved from the left-half to the right-half of the complex plane, which suggests the system gradually loses its stability. In addition, the similar observation can also be obtained from Fig. 5 based on the results of electromagnetic transient simulation. In this figure, when $k$ is increased from 0.80 to 1.20 , the oscillation becomes divergent in the system following the disturbance. This indicates the system gradually loses its stability when $k$ is increased from 0.80 to 1.20 . The consistent observations between the modal analysis and electromagnetic transient 
simulation validate the analytical results of the impact of network parameters on the stability of the HMCS-DPLL.
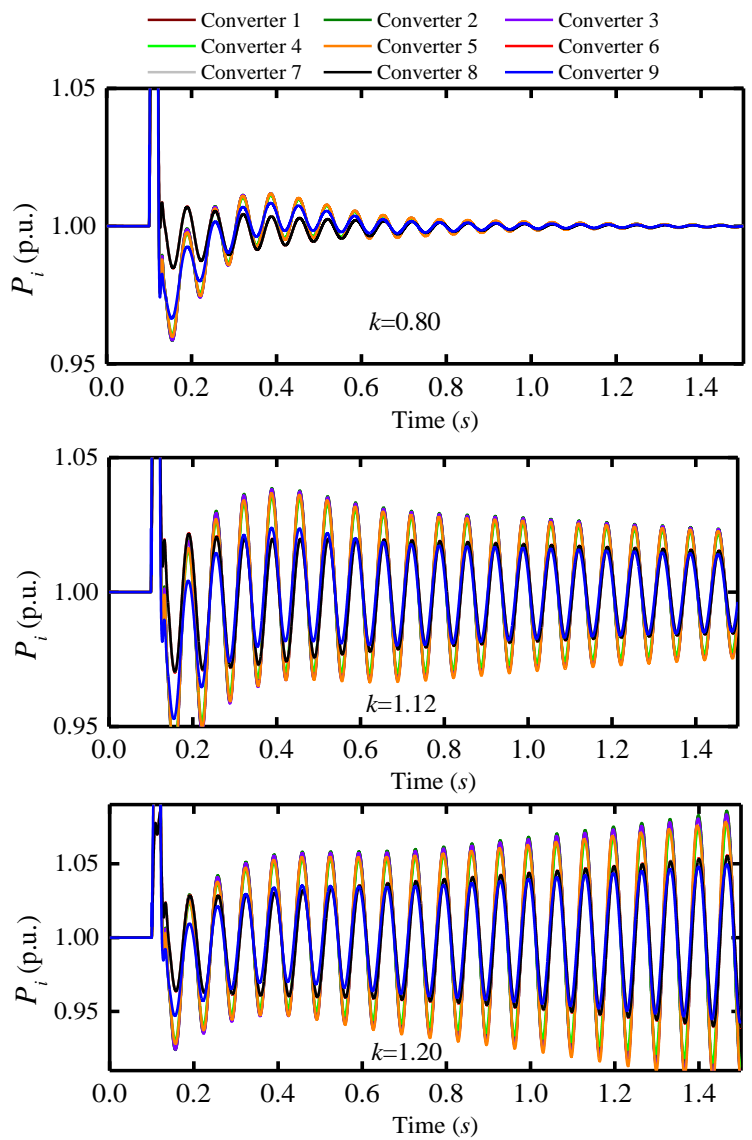

Fig. 5 The active power trajectories of converters in the 9-converter heterogeneous system following a disturbance when $k$ is set as $0.08,1.12$, and 1.20 .

\section{3) Case 3: Impact of converter operating conditions}

In this case, the analytical results of the impact of converter operating conditions on the stability of the HMCS-DPLL are verified on the 9-converter heterogeneous system with the given network parameters and PLL control parameters. The network is the same as the original power network used in the IEEE 39-bus system [23]. The PLL PI control parameters of each converter are set as the first group (1) presented in Table III. Thus, the resulting $K_{\mathrm{I}_{\Sigma}} / K_{\mathrm{P}_{\Sigma} \Sigma}$ in (20) is 551.69. When $P_{0}$ in (20) is set as $0.80,1.10$, and 1.30 , it changes the stability boundary value $K_{c}$ in (20), and the resulting $K_{c}$ is 780.30 , 567.47 , and 480.15. Table VIII presents the values of $K_{c}$ and the dominant eignevalues of the system evaluated based on the fullorder model in (1) and (2) and the reduced-order model in (19), when $P_{0}$ is set as $0.80,1.10$, and 1.30. Also, a disturbance is applied to this system when $P_{0}$ is set as $0.80,1.10$, and 1.30, respectively, for the electromagnetic time-domain simulation using MATLAB/Simulink. The disturbance is the same as the one used in case 1). Fig. 6 shows the active power trajectories of all converters in the 9-converter heterogeneous system following the disturbance when $P_{0}$ is set as $0.80,1.10$, and 1.30 .

It can be observed from Table VIII and Fig. 6 that the system loses its stability when increasing converter power injection $P_{0}$. In Table VIII, when $P_{0}$ is increased from 0.80 to $1.30, K_{c}$ in (20) is reduced from 780.30 to 480.15 . Since $K_{\mathrm{I}_{\Sigma}} / K_{\mathrm{P}_{\Sigma}}$ in (20) is 551.69 , the system loses its stability according to the stability boundary condition in (20) when $P_{0}$ is increased from 0.80 to 1.30. Meanwhile, when $P_{0}$ is increased from 0.80 to 1.30 , the dominant eigenvalues evaluated based on the full-order model and the reduced-order model are also moved from the left-half to the right-half of the complex plane, which suggests the system gradually loses its stability. In addition, the similar observation can also be obtained from Fig. 6 based on the results of electromagnetic transient simulation. In this figure, when $P_{0}$ is increased from 0.80 to 1.30 , the oscillation becomes divergent in the system following the disturbance. This indicates the system gradually loses its stability when $P_{0}$ is increased from 0.80 to 1.30. The observations from electromagnetic transient simulation agrees with modal analysis, which validates the analytical results of the impact of converter operating conditions on the stability of the HMCSDPLL.

Table VIII The dominant eigenvalues of the 9-conveter system evaluated based on the full-order model and the reduced-order model when $P_{0}$ is set as 0.80 , 1.10 , and 1.30 .
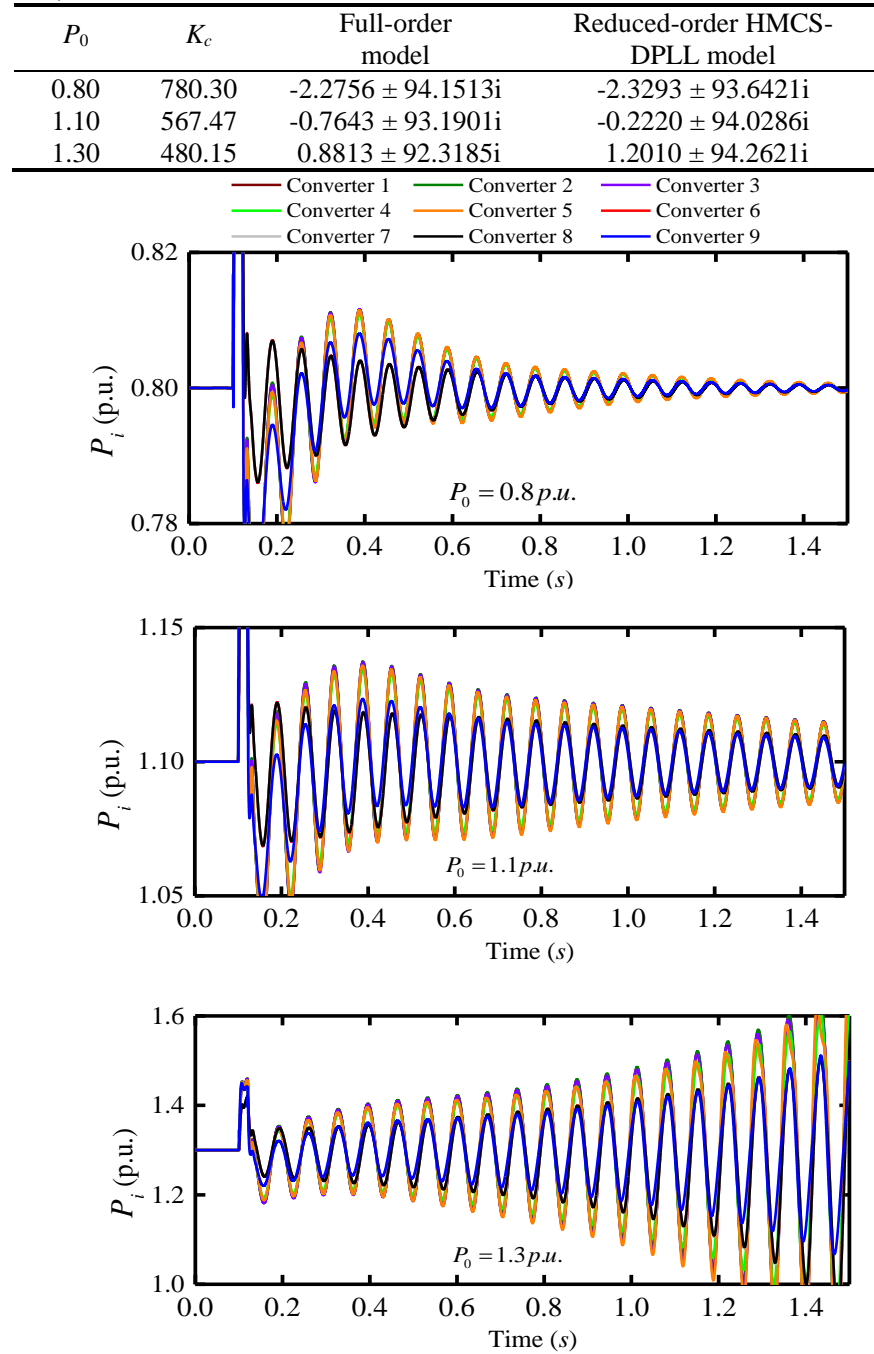

Fig. 6 The active power trajectories of converters in the 9-converter heterogeneous system following a disturbance when $\mathrm{P}_{0}$ is set as $0.80,1.10$, and 1.30 . 


\section{V.CONCLUSION}

In this paper, we analytically derived the small-signal stability boundary of the HMCS-DPLL to understand how the PLL dynamics collectively affect the stability of the HMCS. Based on the derived stability boundary condition, the analytical conclusions were obtained to explain how the stability of the HMCS-DPLL is affected by network connection, converter operating conditions, PLL control parameters, and dynamic interaction among different PLLs. Also, a computationally efficient method was proposed to identify the small-signal stability and stability margin of the HMCS-DPLL as well as the design rationality of PLL control parameters. The derived stability boundary condition, and the analytical conclusions, and the proposed method are validated by modal analysis and electromagnetic transient simulation with detailed models on a 9-converter heterogeneous system. The analytical conclusions and the proposed method are useful to guide the grid planning to reduce and even mitigate the instability risk in the HMCS-DPLL.

\section{REFERENCES}

[1] D. Dong, B. Wen, D. Boroyevich, et al., "Analysis of phase-locked loop low-frequency stability in three-phase grid-connected power converters considering impedance interactions," IEEE Trans. Ind. Electron., vol. 62, no. 1, pp. 310-321, Jan. 2015.

[2] J. Z. Zhou, H. Ding, S. Fan, et al., "Impact of short-circuit ratio and phaselocked-loop parameters on the small-signal behavior of a VSC-HVDC converter," IEEE Trans. Power Del., vol. 29, no. 5, pp. 2287-2296, Oct. 2014.

[3] L. Huang, H. Xin, Z. Li, et al., "Grid-synchronization stability analysis and loop shaping for PLL-based power converters with different reactive power control", IEEE Trans. Smart Grid, vol. 11, no. 1, pp. 501-516, Jan. 2020

[4] L. Fan, "Modeling type-4 wind in weak grids," IEEE Trans. Sustain. Energy, vol. 10, no. 2, pp. 853-864, Apr. 2019.

[5] L. Fan and Z. Miao, "An explanation of oscillations due to wind power plants weak grid interconnection," IEEE Trans. Sustain. Energy, vol. 9, no.1, pp. 488-490, Jan. 2018.

[6] B. Wen, D. Dong, D. Boroyevich, R. Burgos, P. Mattavelli and Z. Shen, "Impedance-based analysis of grid-synchronization stability for threephase paralleled converters", IEEE Trans. Power Electron., vol. 31, no. 1, pp. 26-38, Jan. 2016.

[7] Du W, Dong W, Wang H, "A method of reduced-order modal computation for planning grid connection of a large-scale wind farm," IEEE Trans. Sustain. Energy, vol. 11, no. 3, pp. 1185-1198, July 2020.

[8] D. H. R. Suriyaarachchi, U. D. Annakkage, C. Karawita, and U. D. Annakkage, "A procedure to study sub-synchronous interactions in wind integrated power system," IEEE Trans. Power Syst., vol. 28, no. 1, pp. 377-384, Jul. 2012.

[9] L. P. Kunjumuhammed, B. C. Pal, C. Oates, et al., "Electrical oscillations in wind farm systems: analysis and insight based on detailed modeling," IEEE Trans. Sustain. Energy, vol. 7, no. 1, pp. 51-62, Jan. 2016.

[10] L. Piyasinghe, Z. Miao, J. Khazaei and L. Fan, "Impedance model-based SSR analysis for TCSC compensated type-3 wind energy delivery systems," IEEE Trans. Sustain Energy, vol. 6, no. 1, pp. 179-187, Jan. 2015.

[11] L. Harnefors, M. Bongiorno and S. Lundberg, "Input-admittance calculation and shaping for controlled voltage-source converters," IEEE Trans. Ind. Electron., vol. 54, no. 6, pp. 3323-3334, Dec. 2007.

[12] J. Sun, "Impedance-based stability criterion for grid-connected inverters," IEEE Trans. Power Electron., vol. 26, no. 11, pp. 3075-3078, Nov. 2011.

[13] W. Du, X. Chen and H. Wang, "A method of open-loop modal analysis to examine the SSOs in a multi-machine power system with multiple variable-speed wind generators," IEEE Trans. Power Syst., vol. 33, no. 4, pp. 4297-4307, July 2018.

[14] Y. Huang, X. Yuan, J. Hu, et al, "Modeling of VSC connected to weak grid for stability analysis of DC-link voltage control," IEEE J. Emerg. Sel. Topics Power Electron., vol. 3, no. 4, pp. 1193-1204, Dec. 2015.
[15] W. Du, X. Chen, and H. Wang, "PLL-induced modal resonance of gridconnected PMSGs with the power system electromechanical oscillation modes," IEEE Trans. Sustain. Energy, vol. 8 no. 4, pp. 1581-1591, Apr. 2017.

[16] Y. Zhou, L. Zhao and W. Lee, "Robustness analysis of dynamic equivalent model of DFIG wind farm for stability study," IEEE Trans. Ind. Appl., vol. 54, no. 6, pp. 5682-5690, Nov. 2018.

[17] M. Ali, I. IIie, J. Milanovic, et al., "Wind farm model aggregation using probabilistic clustering," IEEE Trans. Power Syst., vol. 28, no. 1, pp. 309316, Feb. 2013.

[18] W. Dong, H. Xin, D. Wu and L. Huang, "Small signal stability analysis of multi-infeed power electronic systems based on grid strength assessment," IEEE Trans. Power Syst., vol. 34, no. 2, pp. 1393-1403, March. 2019.

[19] Huang L, Xin H, Li Z, et al. "Identification of generalized short-circuit ratio for on-line stability monitoring of wind farms," IEEE Trans. Power Syst., vol. 35, no. 4, pp. 3282-3285, July 2020.

[20] W. Du, W. Dong and H. Wang, "Small-signal stability limit of a gridconnected PMSG wind farm dominated by the dynamics of PLLs" IEEE Trans. on Power Syst., Vol. 35, No. 3, pp. 2093-2107, May 2020

[21] G. W. Stewart, "Matrix perturbation theory," 1990.

[22] Golnaraghi F, Kuo B. Automatic control system $10^{\text {th }}$ edition[M]. New York: McGraw Hill Education, 2017: 635-645.

[23] IEEE PES task force on benchmark systems for stability controls, November 19, 2013. Available: http://www.sel.eesc.usp.br/ieee/

\section{APPENDIX A}

\section{Detailed proof of Equation (3)}

The linearized dynamics model of the PLL in Fig. 1 can be expressed as:

$$
\begin{aligned}
\frac{d \Delta x_{1, i}}{d t} & =k_{i, i} \Delta U_{q, i} \\
\frac{d \Delta \theta_{p l l, i}}{d t} & =k_{p, i} \Delta U_{q, i}+\Delta x_{1, i}
\end{aligned}
$$

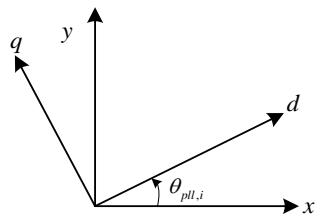

Fig. A1 The relationship between the local $d-q$ and the global $x$-y coordinates

Fig.A1 shows the position relationship between $d q$ local coordinate system used in the vector control of the converters and $x y$ global coordinate system of the power grid. Then, it exists the following relationships:

$$
\begin{gathered}
{\left[\begin{array}{l}
U_{x, i} \\
U_{y, i}
\end{array}\right]=\left[\begin{array}{cc}
\cos \theta_{p l l, i} & -\sin \theta_{p l l, i} \\
\sin \theta_{p l l, i} & \cos \theta_{p l l, i}
\end{array}\right]\left[\begin{array}{l}
U_{d, i} \\
U_{q, i}
\end{array}\right]} \\
{\left[\begin{array}{l}
I_{x, i} \\
I_{y, i}
\end{array}\right]=\left[\begin{array}{cc}
\cos \theta_{p l l, i} & -\sin \theta_{p l l, i} \\
\sin \theta_{p l l, i} & \cos \theta_{p l l, i}
\end{array}\right]\left[\begin{array}{l}
I_{d, i} \\
I_{q, i}
\end{array}\right]}
\end{gathered}
$$

where $U_{d, i}+j U_{q, i}, I_{d, i}+j I_{q, i}$ represent the terminal voltage and output current of $i^{\text {th }}$ converter in the local $d$ - $q$ coordinate, respectively.

Further, the linearization equation corresponding to (23) can be elaborated as:

$$
\Delta U_{q}=\frac{U_{x, i 0}}{U_{i 0}} \Delta U_{y, i}-\frac{U_{y, i 0}}{U_{i 0}} \Delta U_{x, i}-U_{i 0} \Delta \theta_{p l l, i}
$$

As for the single grid-connected converter, its bandwidth of the current inner loop is generally much larger than [20] that of the PLL. Thus, it can be inferred that the converter has perfect current tracking effect which can be modeled as a constant current source $I_{d, i}+j I_{q, i}$ in its own $d-q$ coordinate during the small-signal stability process dominated by PLL. Then,

$$
\Delta I_{d, i}+j \Delta I_{q, i}=0
$$

By combining (24) and (26), we can obtain the linearization equation: 


$$
\begin{aligned}
\Delta I_{x, i} & =-I_{y, i 0} \Delta \theta_{p l l, i} \\
\Delta I_{y, i} & =I_{x, i 0} \Delta \theta_{p l l, i}
\end{aligned}
$$

Then, by combining (22), (25), and (27), the reduced-order linearized model of the $i^{\text {th }}$ converter under the capacity base of the system can be formulated in the following state space format:

$$
\left\{\begin{array}{l}
\frac{d}{d t} \Delta \boldsymbol{X}_{r, i}=\boldsymbol{A}_{r, i} \Delta \boldsymbol{X}_{r, i}+\boldsymbol{B}_{r, i} \Delta \boldsymbol{U}_{i} \\
\Delta \boldsymbol{I}_{i}=\boldsymbol{C}_{r, i} \Delta \boldsymbol{X}_{r, i}
\end{array}\right.
$$

where

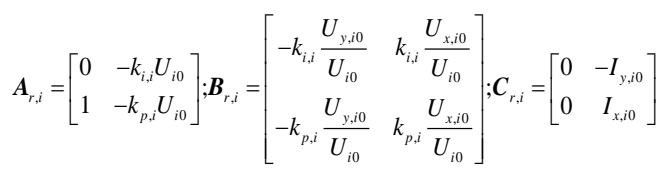

APPENDIX B

\section{Detailed proof of equation (18)}

It is worth mentioning that the steady-state values of all the converters' output current $I_{x, i 0}+j I_{y, i 0}$ can be considered as the same constant value at the planning stage. Thus, the output matrix of each converter satisfies the following relationship: $\boldsymbol{C}_{r, 1}=\boldsymbol{C}_{r, 2}=\ldots=\boldsymbol{C}_{r, n}=\boldsymbol{C}$. On this basis, the detailed derivation procedure of equation (19) can be elaborated as follows:

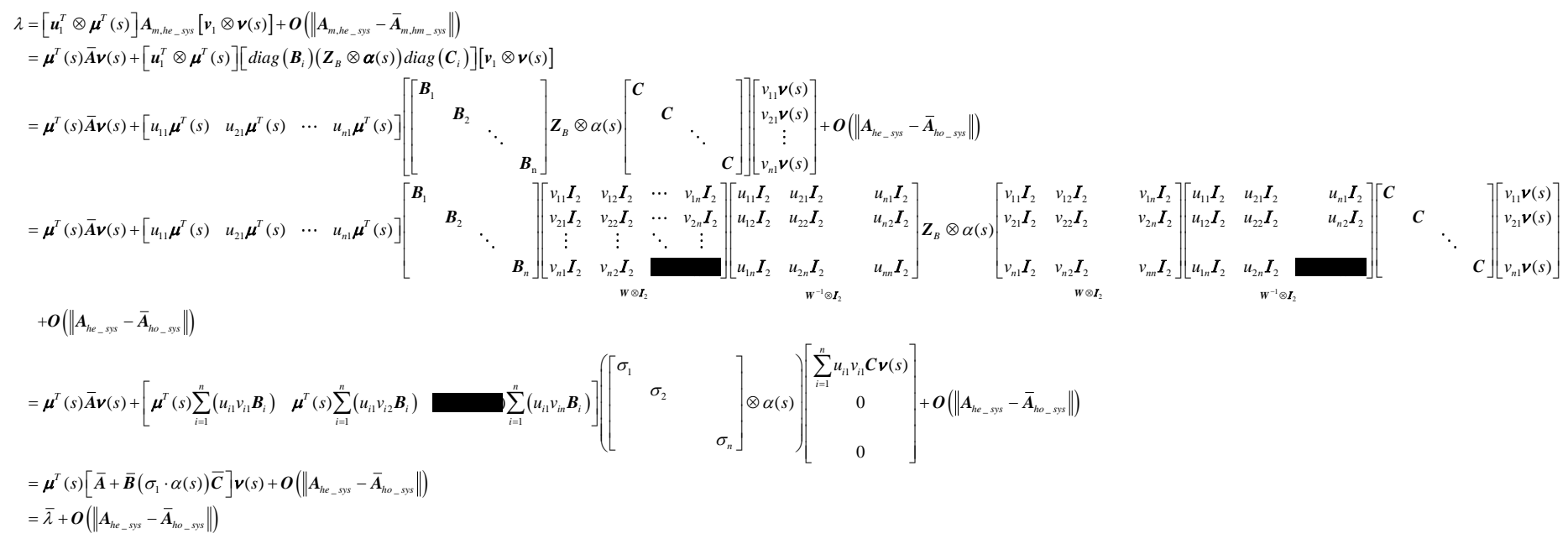

\section{Detailed proof of equation (20)}

APPENDIX C

Since $\bar{A}_{h o_{-} s y s, 1}^{e q}$ in (19) contains Laplace operator $s$, it is tough to solve the characteristic equation (19) directly. For the purpose of eliminating Laplace operator $s$, the state matrix $\overline{\boldsymbol{A}}_{h o_{-} s y s, 1}^{e q}$ needs to be further simplified by:

$$
\begin{aligned}
& \frac{d}{d t} \Delta \boldsymbol{X}_{r}=\overline{\boldsymbol{A}}_{h o_{-} s y, 1}^{e q} \Delta \boldsymbol{X}_{r}, \quad \overline{\boldsymbol{A}}_{h o_{-s y s}}^{e q}=\overline{\boldsymbol{A}}+\overline{\boldsymbol{B}}\left(\sigma_{1} \cdot \alpha(s)\right) \overline{\boldsymbol{C}} \\
& \Rightarrow s\left(\boldsymbol{I}-\frac{\sigma_{1}}{\omega_{0}} \cdot \overline{\boldsymbol{B}} \overline{\boldsymbol{C}}\right) \Delta \boldsymbol{X}_{r}=\left(\overline{\boldsymbol{A}}+\sigma_{1} \cdot \overline{\boldsymbol{B}}\left[\begin{array}{cc}
\tau / \omega_{0} & -1 \\
1 & \tau / \omega_{0}
\end{array}\right] \overline{\boldsymbol{C}}\right) \Delta \boldsymbol{X}_{r} \\
& \Rightarrow \frac{d}{d t} \Delta \boldsymbol{X}_{r}=\overline{\boldsymbol{A}}_{h 0_{-} s s, 1}^{e q} \Delta \boldsymbol{X}_{r}, \\
& \overline{\boldsymbol{A}}_{h o_{-} s y, 1}^{e q}=\left(\boldsymbol{I}-\frac{\sigma_{1}}{\omega_{0}} \overline{\boldsymbol{B}} \overline{\boldsymbol{C}}\right)^{-1}\left(\overline{\boldsymbol{A}}+\sigma_{1} \overline{\boldsymbol{B}}\left[\begin{array}{cc}
\tau / \omega_{0} & -1 \\
1 & \tau / \omega_{0}
\end{array}\right] \overline{\boldsymbol{C}}\right)=\left[\begin{array}{cc}
\frac{\sigma_{1} P_{0} K_{I_{-} \Sigma}}{\omega_{0} U_{0}-\sigma_{1} P_{0} K_{P_{-} \Sigma}} & \frac{\left(\omega_{0} U_{0}-\sigma_{1} P_{0} K_{P_{-} \Sigma}\right)\left(\sigma_{1} Q_{0} \omega_{0} K_{I_{-} \Sigma}-U_{0}^{2} \omega_{0} K_{I_{-} \Sigma}+\sigma_{1} \tau P_{0} K_{I_{-} \Sigma}\right)+\sigma_{1} P_{0} K_{I_{-} \Sigma}\left(\sigma_{1} Q_{0} \omega_{0} K_{P_{-} \Sigma}-U_{0}^{2} \omega_{0} K_{P_{-} \Sigma}+\sigma_{1} \tau P_{0} K_{P_{-} \Sigma}\right)}{\omega_{0} U_{0}\left(\omega_{0} U_{0}-\sigma_{1} P_{0} K_{P_{-} \Sigma}\right)} \\
\frac{\omega_{0} U_{0}}{\omega_{0} U_{0}-\sigma_{1} P_{0} K_{P_{-} \Sigma}} & \frac{-U_{0}^{2} \omega_{0} K_{P_{-} \Sigma}+\sigma_{1} Q_{0} \omega_{0} K_{P_{-} \Sigma}+\sigma_{1} \tau P_{0} K_{P_{-} \Sigma}}{\omega_{0} U_{0}-\sigma_{1} P_{0} K_{P_{-} \Sigma}}
\end{array}\right]
\end{aligned}
$$

Based on (29), the characteristic equation (19) can be represented as:

$$
\left|\lambda I_{2}-\overline{\boldsymbol{A}}_{h o_{-} s y s, 1}^{e q}\right|=0
$$

Then, based on Routh-Hurwitz stability criterion [22] and (30), the HMCS-DPLL is stable if and only if the following conditions are satisfied:

$$
\left\{\begin{array}{l}
\sigma_{1}\left(P_{0} K_{I_{-} \Sigma}+Q_{0} \omega_{0} K_{P_{-} \Sigma}+\tau P_{0} K_{P_{-} \Sigma}\right)<U_{0}^{2} \omega_{0} K_{P_{-} \Sigma} \\
\sigma_{1}\left(Q_{0} \omega_{0}+\tau P_{0}\right)<U_{0}^{2} \omega_{0}
\end{array}\right.
$$

Since $P_{0} K_{I_{-} \Sigma}>0$, the condition (1) can be utilized as the stability boundary condition of the HMCS-DPLL which can be further derived as

$$
\frac{K_{I_{-} \Sigma}}{K_{P_{-} \Sigma}}<K_{c}=\frac{U_{0}^{2} \omega_{0}}{\sigma_{1} P_{0}}-\frac{Q_{0} \omega_{0}}{P_{0}}-\tau
$$

